\title{
Genetic recombination and diversity of sapovirus in pediatric patients with acute gastroenteritis in Thailand, 2010-2018
}

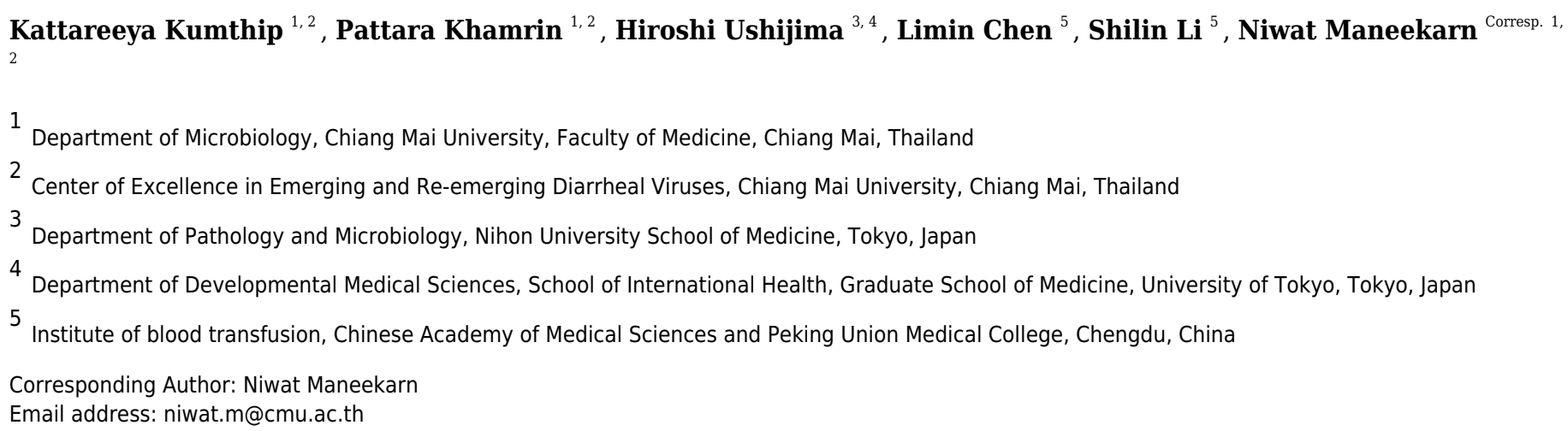

Background. Human sapovirus ( $\mathrm{SaV}$ ) is an etiologic agent of acute gastroenteritis (AGE) in all age groups worldwide. Genetic recombination of SaV has been reported from many countries. So far, none of SaV recombinant strain has been reported from Thailand. This study examined the genetic recombination and genotype diversity of SaV in children hospitalized with AGE in Chiang Mai, Thailand. Methods. Stool samples were collected from children suffering from diarrhea who admitted to the hospitals in Chiang Mai, Thailand between 2010 and 2018. SaV was detected by RT-PCR and the polymerase and capsid gene sequences were analysed. Results. From a total of 3057 samples tested, 50 (1.6\%) were positive for SaV. Among positive samples, SaV genotype GI.1 was the most predominant genotype (40\%; 20/50), followed by GII.1 and GII.5 (each of 16\%; 8/50), GI.2 (14\%; 7/50), GIV.1 (4\%; 2/50), and GI.5 (2\%; 1/50). In addition, 4 SaV recombinant strains of GII.1/GII.4 were identified in this study (8\%; 4/50). Conclusions. The data revealed the genetic diversity of SaV circulating in children with AGE in Chiang Mai, Thailand during 2010 to 2018 and the intragenogroup SaV recombinant strains were reported for the first time in Thailand. 


\section{Genetic recombination and diversity of sapovirus in pediatric patients with}

\section{2 acute gastroenteritis in Thailand, 2010-2018}

3

4 Kattareeya Kumthip ${ }^{1,2}$, Pattara Khamrin ${ }^{1,2}$, Hiroshi Ushijima ${ }^{3,4}$, Limin Chen ${ }^{5}$, Shilin Li $^{5}$, Niwat

5 Maneekarn ${ }^{1,2}$

$7{ }^{1}$ Department of Microbiology, Faculty of Medicine, Chiang Mai University, Chiang Mai, 8 Thailand

$9{ }^{2}$ Center of Excellence in Emerging and Re-emerging Diarrheal Viruses, Chiang Mai University, 10 Chiang Mai, Thailand,

$11{ }^{3}$ Department of Developmental Medical Sciences, School of International Health, Graduate

12 School of Medicine, The University of Tokyo, Tokyo, Japan

$13{ }^{4}$ Division of Microbiology, Department of Pathology and Microbiology, Nihon University

14 School of Medicine, Tokyo, Japan,

$15{ }^{5}$ Institute of blood transfusion, Chinese Academy of Medical Sciences and Peking Union

16 Medical College, Chengdu, China

Corresponding Author:

20 Niwat Maneekarn ${ }^{1,2}$

21110 Inthawarorot Rd, Sri Phum, Muang Chiang Mai, Chiang Mai 50200, Thailand

22 Email address: niwat.m@cmu.ac.th 


\section{Abstract}

27 Background. Human sapovirus $(\mathrm{SaV})$ is an etiologic agent of acute gastroenteritis (AGE) in all age groups worldwide. Genetic recombination of $\mathrm{SaV}$ has been reported from many countries. So far, none of $\mathrm{SaV}$ recombinant strain has been reported from Thailand. This study examined the genetic recombination and genotype diversity of $\mathrm{SaV}$ in children hospitalized with AGE in

\section{Chiang Mai, Thailand.}

32 Methods. Stool samples were collected from children suffering from diarrhea who admitted to the hospitals in Chiang Mai, Thailand between 2010 and 2018. SaV was detected by RT-PCR and the polymerase and capsid gene sequences were analysed.

Results. From a total of 3,057 samples tested, 50 (1.6\%) were positive for $\mathrm{SaV}$. Among positive samples, $\mathrm{SaV}$ genotype GI.1 was the most predominant genotype $(40 \% ; 20 / 50)$, followed by GII.1 and GII.5 (each of 16\%; 8/50), GI.2 (14\%; 7/50), GIV.1 (4\%; 2/50), and GI.5 (2\%; 1/50). In addition, $4 \mathrm{SaV}$ recombinant strains of GII.1/GII.4 were identified in this study $(8 \%$; $4 / 50)$.

Conclusions. The data revealed the genetic diversity of $\mathrm{SaV}$ circulating in children with AGE in reported for the first time in Thailand. 
Introduction

Sapovirus $(\mathrm{SaV})$ is one of the important pathogens that cause outbreaks and sporadic cases of acute gastroenteritis (AGE) in people of all ages worldwide (Torner et al. 2016). Prevalences of $\mathrm{SaV}$ infection have been reported between $0.2 \%$ and $39 \%$ in children with AGE (Magwalivha et al. 2018) and between 2.2\% and 15.6\% in all age groups (Oka et al. 2015; Varela et al. 2019). SaV belongs to genus Sapovirus of the Caliciviridae family. SaV particle is a - small, non-enveloped with $30-35 \mathrm{~nm}$ in diameter. $\mathrm{SaV}$ has a positive-sense, single stranded RNA genome of about 7.1-7.7 $\mathrm{kb}$ in length which contains two open reading frames (ORFs) (Green 2013; Oka et al. 2016). ORF1 encodes for nonstructural proteins (NS1, NS2, NS3, NS4, NS5, and NS6-NS7) and the major capsid protein (VP1). ORF2 encodes for a minor capsid protein (VP2) (Oka et al. 2015). Based on the entire nucleotide sequence of VP1 region, SaV can be classified into 19 genogroups (GI-GXIX) and only four genogroups (GI, GII, GIV, and GV) known to infect human (Yinda et al. 2017). Human $\mathrm{SaV}$ can be further divided into 18 genotypes 62 (GI.1 to GI.7, GII.1 to GII.8, GIV.1, GV.1, and GV.2) (Kagning Tsinda et al. 2017; Liu et al. 63 2016; Oka et al. 2012; Oka et al. 2015; Xue et al. 2019) with one additional genotype of GII.NA 64 that has been reported recently (Diez-Valcarce et al. 2019). Among these, SaV GI and GII are 65 the most prevalent genogroups detected worldwide while other genogroups have been rarely 66 detected (Magwalivha et al. 2018). Like noroviruses, several SaV recombinant strains of both 67 intragenogroup and intergenogroup recombinations have been reported (Chanit et al. 2009; 68 Lasure \& Gopalkrishna 2017; Liu et al. 2015). Within SaV genome, recombination generally 69 occurs in the ORF1, particularly at the junction between the polymerase (NS7) and capsid (VP1) 
70 genes (Hansman et al. 2005; Katayama et al. 2004; Phan et al. 2006). Some of SaV recombinant

71 strains have been documented to associate with the outbreaks (Hansman et al. 2007; Lee et al.

72 2012). In Thailand, SaV infection in children with diarrhea has been reported previously,

73 however, none of $\mathrm{SaV}$ recombinant strain has been reported previously. Therefore, this study

74 aimed to investigate the genetic recombination and genotype diversity of $\mathrm{SaV}$ circulating in

75 children hospitalized with AGE in Chiang Mai, Thailand from 2010 to 2018.

76

77

78

79

80

81

82 83

84

85

86

87

88

89

90

91

92

\section{Materials \& Methods}

\section{Sample collection}

Stool samples were collected from children who admitted to hospitals with AGE during the period 2010 to 2018 from five major hospitals in Chiang Mai province, northern Thailand, including Maharaj Nakorn Chiang Mai Hospital, Sriphat Medical Center, Nakornping Hospital, Sanpatong Hospital, and Rajavej Chiang Mai Hospital. The age of patients enrolled in this study ranged from neonate to 15 years old. Acute gastroenteritis was defined by watery diarrhea with three or more stool episodes per day for less than 14 days (Green. 2013). All specimens were stored at $-20^{\circ} \mathrm{C}$ until investigation. This work was conducted under the approval of the Research Ethics Committee of the Faculty of Medicine, Chiang Mai University (MIC-2557-02710). The written informed consent form was obtained from parents before samples were collected from their children

\section{Detection of SaV by RT-PCR}

Viral nucleic acid was extracted from $200 \mu$ of the supernatant of a $10 \%$ stool suspension prepared in phosphate-buffered saline ( $\mathrm{pH}$ 7.4) using the Geneaid Viral Nucleic Acid Extraction 
93 Kit II (Geneaid, Taiwan) according to the manufacturer's protocol. The viral RNA was reverse

94 transcribed to cDNA using random hexamer primers and RevertAid ${ }^{\mathrm{TM}}$ reverse transcriptase

95 (Thermo Fisher Scientific, USA) according to the manufacturer's instruction. SaV was screened

96 by conventional PCR method using GoTaq DNA polymerase (Promega, USA) with primers

97 SLV5731 and SLV5749 (Table 1) targeting capsid region (VP1 gene) as described previously

98 (Yan et al. 2003). The PCR cycling condition was as follows: initial denature at $94^{\circ} \mathrm{C}$ for 3 min,

9935 cycles step of denature at $94^{\circ} \mathrm{C}$ for $1 \mathrm{~min}$, anneal at $58^{\circ} \mathrm{C}$ for $1 \mathrm{~min}$, and extend at $72^{\circ} \mathrm{C}$ for 1

$100 \mathrm{~min}$, followed by final extension at $72{ }^{\circ} \mathrm{C}$ for $10 \mathrm{~min}$. Amplicon size of $434 \mathrm{bp}$ was separated on

$101 \quad 1.5 \%$ agarose gel electrophoresis and stained with nucleic acid staining solution (RedSafe,

102 INtRON Biotechonology, South Korea) before subjecting to visualize under ultraviolet

103 transilluminator. In addition to $\mathrm{SaV}$ screening, the same set of stool specimens were also tested

104 for several other diarrhea-causing viruses, including rotavirus, norovirus, astrovirus, adenovirus,

105 enterovirus, parechovirus, and Aichivirus using the protocol described previously (Khamrin et al.

106 2011).

107 Sequence analysis and genotype identification

108 A Gel/PCR DNA Fragment Extraction Kit (Geneaid, Taiwan) was used to purify

109 amplicons of SaV-capsid gene according to the manufacturer's protocol. All purified PCR

110 products were direct sequenced using Applied Biosystems BigDye ${ }^{\circledR}$ Terminator Cycle

111 Sequencing Kit v3.1 (Life Technologies, USA) with forward and reverse primers SLV5731 and

112 SLV5749, and analyzed by using Applied Biosystems 3100 Genetic Analyzer (Life

113 Technologies, USA). Nucleotide sequences of partial capsid gene were analyzed manually using

114 BioEdit and Clustal X softwares. Identification of virus genotype was initially determined by

115 using the BLAST Tool (https://blast.ncbi.nlm.nih.gov/Blast.cgi) and Human Calicivirus Typing 
116 Tool (https://norovirus.ng.philab.cdc.gov/) and confirmed by phylogenetic analysis using

117 MEGA7 software package (Kumar et al. 2016). The tree was statistically supported by

118 bootstrapping with 1000 replicates.

119 Recombination analysis

120 To examine genetic recombination of $\mathrm{SaV}$ strains detected in this study, the polymerase 121 (RdRp) region was amplified by using primers shown in Table 1. The PCR amplification was 122 carried out under the same condition as used for the VP1 amplification except for the annealing 123 step which was performed at $45^{\circ} \mathrm{C}$ for $1 \mathrm{~min}$. Amplicon sizes of the amplified RdRp region were 124 varied depending on the used primers (746 bp, 824 bp, 827 bp, or 905 bp) shown in Table 1 125 (Berke et al. 1997; Harada et al. 2013; Honma et al. 2001; Martinez et al. 2002). Phylogenetic 126 tree of the partial capsid gene sequences was constructed by using the Maximum Likelihood 127 method based on the Kimura 2-parameter model. Phylogenetic tree of the partial RdRp region 128 was inferred by using the Maximum Likelihood method based on the General Time Reversible 129 model. To predict the putative recombination point for $\mathrm{SaV}$ recombinant strains, nucleotide 130 sequences of the polymerase and capsid genes, spanning the RdRp-VP1 junction region 131 (positions 5078-5265 according to SaV genome of accession no. X86560), were analyzed. The 132 possible recombination point of $\mathrm{SaV}$ strain was determined by using SimPlot software v.3.5.1 133 (Lole et al. 1999). In addition, the confidence interval for recombination between the query 134 strain and parent strains was examined by the Recombination Detection Program v.4.71 (RDP4) 135 complemented with the Max-Chi test to confirm the significant events $(p<0.01)$ (Martin et al. 136 2015).

\section{$137 \quad$ Nucleotide accession number}


141 respectively.

\section{Results}

Prevalence of SaV

A total of 3,057 stool samples collected between 2010 and 2018 were screened for the

presence of $\mathrm{SaV}$. The average of $\mathrm{SaV}$ detection rate over the study period of nine years was $1.6 \%$ (50 out of 3057) (Table 1). From 2010 to 2015, $\mathrm{SaV}$ was detected at low prevalence (0-1.8\%).

After that, the prevalence of $\mathrm{SaV}$ infection increased year by year to $2.2 \%, 2.5 \%$, and $2.8 \%$ in observed in this study. The age of SaV-infected patients varied from 8 months up to 11 years (Table 2). Among $50 \mathrm{SaV}$ positive cases, 26 (52\%) were male and 24 (48\%) were female. All

$157 \mathrm{SaV}$ positive samples were also tested for the presence of other enteric viral pathogens. The results showed that majority of cases were single infection with $\mathrm{SaV}$ (74\%, 37 out of 50) whereas the rest of cases $(26 \%, 13$ out of 50$)$ were co-infected with other enteric viruses including rotavirus, norovirus, adenovirus, parechovirus, enterovirus, or astrovirus (Table 3). 
161

162

163

164

165

166

167

168

169

170

171

172

173

174

175

176

177

178

179

180

181

182

183

\section{Molecular characterization of SaV and phylogenetic analysis}

The partial capsid gene sequence (308 nucleotides) of $50 \mathrm{SaV}$ strains were analyzed to identify genotype of the virus. Seven different genotypes were identified in the present study: GI.1 (20), GI.2 (7), GI.5 (1), GII.1 (8), GII.4 (4), GII.5 (8), and GIV.1 (2). Phylogenetic analysis revealed that $20 \mathrm{SaV}$ GI.1 strains were clustered with the GI.1 strains reported previously from USA, Korea, Japan, and Brazil (Fig. 1) and showed 97.4-99.6\% nucleotide homology to theses strains. Seven SaV GI.2 strains were grouped together with the GI.2 strains reported previously from Brazil and USA with highest nucleotide sequence similarity (98.7-100\%). GI.5 strain was closely related to the GI.5 Ehime643 strain detected previously in Japan $(99.0 \%$ nucleotide sequence identity). Eight GII.1 strains shared high similarity (90.3-95.0\% nucleotide sequence identities) with GII.1 strains detected previously in United Kingdom, USA, India, Japan, and Thailand. Four GII.4 strains were closely related to GII.4 strains reported from Philippines and Peru (94.5-97.1\% nucleotide identities). All GII.5 strains shared highest similarity (90.3-95.0\%) with the GII.5 SataRosa3693/2015/GT strain detected in Guatemala. The CMH-N061-12 SaV GIV.1 strain displayed 97.9-99.6\% nucleotide identities to GIV.1 strains reported previously from USA and Japan while CMH-N028-18 SaV GIV.1 strain shared 87.1-87.8\% sequence homology with those of the SaV GIV.1 reference strains.

\section{Recombination analysis}

In the $\mathrm{SaV}$ genome, recombination event typically occurs between the polymerase (RdRp) and capsid (VP1) genes. To investigate the genetic recombination of $\mathrm{SaV}$ strains detected in this study, we further amplified the polymerase gene of the viruses. The partial RdRp gene sequence (745 nucleotides) was successfully obtained from 49 of $50 \mathrm{SaV}$-positive samples. Phylogenetic tree of the partial RpRp region of $49 \mathrm{SaV}$ strains was constructed (Fig. 2) and the 
184 results showed that all strains, except for four GII.4 strains (CMH-S057-17, CMH-S050-17, 185 CMH-N021-13, and CMH-N145-12), were clustered into the same genotypes assigned by the 186 capsid gene sequence as shown previously in Figure 1. Based in the capsid gene sequence (Fig. 187 1), CMH-S057-17, CMH-S050-17, CMH-N021-13, and CMH-N145-12 were assigned as the 188 GII.4 genotype whereas based on the RdRp sequence they clustured together with SaV GII.1 189 reference strains and shared 91.8-95.0\% nucleotide sequence identities. The data suggested that 190 these four SaV strains were the SaV GII.1/GII.4 recombinant strains. To predict the putative 191 recombination point for SaV recombinant strains, SimPlot software was used. The similarity plot 192 of SaV recombinant strains detected in this study are shown in Figure 3. Two recombination 193 break points were identified at positions 5088 and 5091 within the RdRp-VP1 junction region 194 (nucleotide positions 5078-5265). The CMH-145-12 and CMH-N021-13 strains showed the 195 same recombination point at position $5088\left(p=1.572 \times 10^{-14}\right.$ and $p=1.496 \times 10^{-12}$, respectively) 196 while other 2 strains (CMH-S050-17 and CMH-S057-17) displayed the recombination point at 197 position $5091\left(p=2.334 \times 10^{-14}\right.$ and $p=2.322 \times 10^{-14}$, respectively).

\section{SaV infection in different age groups of patients}

Among $\mathrm{SaV}$ positive cases, the highest detection rate was seen in patients with the age of 2 to $<3$ years $(40.5 \%)$, followed by 1 to $<2$ years $(35.7 \%)$, less than 1 year $(9.5 \%), 3$ to $<4$ years

$201(7.1 \%), 4$ to $<5$ years $(4.8 \%)$, and more than 5 years $(2.4 \%)$. In addition, this study identified 6 202 different genotypes and one recombinant pattern of $\mathrm{SaV}$. Distribution of $\mathrm{SaV}$ genotypes detected 203 in different age groups of patients is shown in Figure 4. It was found that GI.1 genotype was 204 detected in all age groups except for patients with more than 5 years of age. The GII.5 was also 205 identified in patients with age groups of less than 4 years. Interestingly, recombinant $\mathrm{SaV}$ 206 GII.1/GII.4 was detected in patients between 1 and 2 years of age. 


\section{Discussion}

221 of human in all age group worldwide (Khamrin et al. 2017; Lu et al. 2014; Neo et al. 2017; Sala 222 et al. 2014). The detection rate of SaV infection in many countries around the world have been 223 reported with a range from 0.2 to $39 \%$ in children and 2.2 to $15.6 \%$ in all age groups 224 (Magwalivha et al. 2018; Oka et al. 2015; Varela et al. 2019). In Thailand, the prevalence of SaV 225 was detected between $0 \%$ and $15.0 \%$ (Kumthip \& Khamrin 2018). The overall SaV-positive rate 226 reported in the present study during the period 2010 to $2018(1.6 \%$, ranged from 0 to $2.8 \%)$ is 227 more or less the same as other similar studies including Bangladesh (2.7\%) (Dey et al. 2007), 228 China (1.9\%) (Ren et al. 2013), Vietnam (1.4\%) (Trang et al. 2012), and Thailand (1.2 and 1.9\%) 229 (Khamrin et al. 2017; Supadej et al. 2019). However, when compared to the studies conducted in 
230 different geographical regions, the prevalence of $\mathrm{SaV}$ detected in this study was lower than those 231 reported from Japan (4.8\%), Philippines (7\%), USA (5.4\%), Italy (6\%), Denmark (8.8\%),

232 Finland (9.3\%), Spain (15.6\%), and Nicaragua (17\%) (Biscaro et al. 2018; Bucardo et al. 2014;

233 Chhabra et al. 2013; Oka et al. 2015; Thongprachum et al. 2015; Varela et al. 2019). The

234 variation of $\mathrm{SaV}$ prevalence in different studies could be explained, at least in part, by the

235 difference in study locations, the detection methods, and the emergence of new epidemic strains.

236 It was noticed that our study as well as other studies that had similar $\mathrm{SaV}$ prevalence used single

237 round PCR as a screening method while other studies that reported higher SaV-positive rate 238 performed real-time PCR for the screening process.

Among different genogroups of SaV, the SaV GI (GI.1 and GI.2) and GII (GII.1 and

GII.2) are the most predominant genogroups circulating worldwide while other genotypes are

rarely detected in some particular countries (Diez-Valcarce et al. 2018; Magwalivha et al. 2018).

242 Similar to other reports, SaV GI.1, GI.2, and GII.1 were the most common genotypes detected in 243 our study. Nonetheless, there was no GII.2 strain observed in the present study. The SaV GII.5 is 244 not often detected. The occurrence of GII.5 in human stool samples was reported in some 245 particular areas such as Guatemala, Peru, South Africa, and USA (Diez-Valcarce et al. 2018; Liu 246 et al. 2016; Murray et al. 2016). In addition to these countries, it should be noted that a high

247 proportion of GII.5 strain (16\%, 8 out of 50) was observed in our study. Interestingly, 7 out of 8 248 SaV GII.5-infected patients were from the same hospital (Sanpatong hospital) and 5 of them 249 were detected in the same year of 2018, suggesting that this particular genotype is circulating in 250 a particular location. virus diversity (Worobey \& Holmes 1999). At least, two types of recombination events of $\mathrm{SaV}$ 
253 including intergenogroup and intragenogroup have been reported previously (Chanit et al. 2009;

254 Hansman et al. 2007; Hansman et al. 2005; Katayama et al. 2004; Oka et al. 2015; Phan et al.

255 2006). In the present study, intragenogroup recombinant GII.1/GII.4 SaV was detected in four

256 samples, accounting for 8\% (4/50) of all SaV infected cases. The recombinant GII.1/GII.4 SaV

257 has been reported previously in Philippines, Vietnam, and USA (Diez-Valcarce et al. 2018; Liu

258 et al. 2015; Nguyen et al. 2008). However, it has not been described elsewhere in Thailand. To

259 our knowledge, this is the first report demonstrating the presence of recombinant $\mathrm{SaV}$ in

260 Thailand. Generally, characterization of $\mathrm{SaV}$ is based on the nucleotide sequence of capsid gene

261 (Oka et al. 2012) and many other previous studies have identified $\mathrm{SaV}$ genotypes based only on

262 this gene. To date, both capsid and polymerase genes are used to classify genotype of

263 noroviruses (Chhabra et al. 2019). Since $\mathrm{SaV}$ is a very similar pathogen in many aspects,

264 therefore, future classification and characterization of $\mathrm{SaV}$ should rely on both polymerase and

265 capsid sequences to identify the virus diversity. In addition, continued surveillance on $\mathrm{SaV}$ is

266 important to monitor the emergence of new virus strains.

\section{Conclusions}

268 In summary, the results of this study highlight the impact of $\mathrm{SaV}$ in diarrheal diseases

269 among children in Chiang Mai, Thailand over the period of nine years and is the first report to

270 describe recombinant $\mathrm{SaV}$ infection in Thai children suffering with AGE. The data of nucleotide

271 analysis of both polymerase and capsid genes from this study provide useful information for a

272 better understanding on the caliciviruses other than noroviruses.

273

274 Acknowledgements 
275 We are grateful to the physicians and nursing staff who helped to collect the specimens

276 used in this study.

277

278

279

280

281

282

283

284

285

286

287

288

289

290

291

292 References

293 Berke T, Golding B, Jiang X, Cubitt DW, Wolfaardt M, Smith AW, and Matson DO. 1997.

294 Phylogenetic analysis of the Caliciviruses. J Med Virol 52:419-424.

295 Biscaro V, Piccinelli G, Gargiulo F, Ianiro G, Caruso A, Caccuri F, De Francesco MA. 2018.

296 Detection and molecular characterization of enteric viruses in children with acute 
297

298

299

300

301

302

303

304

305

306

307

308

309

310

311

312

313

314

315

316

317

318

gastroenteritis in Northern Italy. Infect Genet Evol 60:35-41. DOI

10.1016/j.meegid.2018.02.011

Bucardo F, Reyes Y, Svensson L, Nordgren J. 2014. Predominance of norovirus and sapovirus in Nicaragua after implementation of universal rotavirus vaccination. PLoS One 9(5):e98201. DOI 10.1371/journal.pone.0098201. eCollection 2014

Chanit W, Thongprachum A, Khamrin P, Okitsu S, Mizuguchi M, and Ushijima H. 2009. Intergenogroup recombinant sapovirus in Japan, 2007-2008. Emerg Infect Dis 15:10841087. DOI 10.3201/eid1507.090153

Chhabra P, Payne DC, Szilagyi PG, Edwards KM, Staat MA, Shirley SH, Wikswo M, Nix WA, Lu X, Parashar UD, Vinjé J. 2013. Etiology of viral gastroenteritis in children $<5$ years of age in the United States, 2008-2009. J Infect Dis 208(5):790-800. DOI 10.1093/infdis/jit254

Chhabra P, de Graaf M, Parra GI, Chan MC, Green K, Martella V, Wang Q, White PA, Katayama K, Vennema H, Koopmans MPG, Vinjé J. 2019. Updated classification of norovirus genogroups and genotypes. J Gen Virol 100(10):1393-1406. DOI 10.1099/jgv.0.001318

Dey SK, Phan TG, Nguyen TA, Nishio O, Salim AF, Yagyu F, Okitsu S, and Ushijima H. 2007. Prevalence of sapovirus infection among infants and children with acute gastroenteritis in Dhaka City, Bangladesh during 2004-2005. J Med Virol 79:633-638. DOI $10.1002 / j m v .20859$

Diez-Valcarce M, Castro CJ, Marine RL, Halasa N, Mayta H, Saito M, Tsaknaridis L, Pan CY, Bucardo F, Becker-Dreps S, Lopez MR, Magana LC, Ng TFF, and Vinje J. 2018. Genetic 
321

Diez-Valcarce M, Montmayeur A, Tatusov R, Vinjé J. 2019. Near-Complete Human Sapovirus Genome Sequences from Kenya. Microbiol Resour Announc 8(7). pii: e01602-18. DOI 10.1128/MRA.01602-18. eCollection 2019

Green KY. 2013. Caliciviridae: The noroviruses. In: Knipe DM, Howley P, eds. Field's virology $6^{\text {th }}$ edn. Philadelphia: Lippincott Williams \& Wilkins :584-587

Hansman GS, Ishida S, Yoshizumi S, Miyoshi M, Ikeda T, Oka T, and Takeda N. 2007. Recombinant sapovirus gastroenteritis, Japan. Emerg Infect Dis 13:786-788. DOI 10.3201/eid1305.070049

Hansman GS, Takeda N, Oka T, Oseto M, Hedlund KO, and Katayama K. 2005. Intergenogroup recombination in sapoviruses. Emerg Infect Dis 11:1916-1920. DOI 10.3201/eid1112.050722

Harada S, Tokuoka E, Kiyota N, Katayama K, and Oka T. 2013. Phylogenetic analysis of the nonstructural and structural protein encoding region sequences, indicating successive appearance of genomically diverse sapovirus strains from gastroenteritis patients. Jpn J Infect Dis 66:454-457.

Honma S, Nakata S, Sakai Y, Tatsumi M, Numata-Kinoshita K, and Chiba S. 2001. Sensitive detection and differentiation of Sapporo virus, a member of the family Caliciviridae, by standard and booster nested polymerase chain reaction. J Med Virol 65:413-417.

Kagning Tsinda E, Malasao R, Furuse Y, Gilman RH, Liu X, Apaza S, Espetia S, Cama V, Oshitani H, and Saito M. 2017. Complete Coding Genome Sequences of Uncommon 
341

342

343

344

345

346

347

348

349

350

351

352

353

354

355

356

357

358

359

360

361

GII.8 Sapovirus Strains Identified in Diarrhea Samples Collected from Peruvian Children. Genome Announc 5. 10.1128/genomeA.01137-17

Katayama K, Miyoshi T, Uchino K, Oka T, Tanaka T, Takeda N, and Hansman GS. 2004. Novel recombinant sapovirus. Emerg Infect Dis 10:1874-1876. DOI 10.3201/eid1010.040395

Khamrin P, Kumthip K, Supadej K, Thongprachum A, Okitsu S, Hayakawa S, Ushijima H, and Maneekarn N. 2017. Noroviruses and sapoviruses associated with acute gastroenteritis in pediatric patients in Thailand: increased detection of recombinant norovirus GII.P16/GII.13 strains. Arch Virol 162:3371-3380. DOI 10.1007/s00705-017-3501-3

Khamrin P, Okame M, Thongprachum A, Nantachit N, Nishimura S, Okitsu S, Maneekarn N, Ushijima H. 2011. A single-tube multiplex PCR for rapid detection in feces of 10 viruses causing diarrhea. J Virol Methods 173(2):390-393. DOI 10.1016/j.jviromet.2011.02.012

Kumar S, Stecher G, and Tamura K. 2016. MEGA7: Molecular Evolutionary Genetics Analysis Version 7.0 for Bigger Datasets. Mol Biol Evol 33:1870-1874. DOI 10.1093/molbev/msw054

Kumthip K, and Khamrin P. 2018. Molecular epidemiology and genotype distributions of noroviruses and sapoviruses in Thailand 2000-2016: A review. J Med Virol 90:617-624. DOI 10.1002/jmv.25019

Lasure N, and Gopalkrishna V. 2017. Epidemiological profile and genetic diversity of sapoviruses ( $\mathrm{SaVs}$ ) identified in children suffering from acute gastroenteritis in Pune, Maharashtra, Western India, 2007-2011. Epidemiol Infect 145:106-114. DOI $10.1017 / \mathrm{s} 0950268816001953$ 
362 Lee LE, Cebelinski EA, Fuller C, Keene WE, Smith K, Vinje J, and Besser JM. 2012. Sapovirus

363

364

365

366

367

368

369

370

371

372

373

374

375

376

377

378

379

380

381

382

outbreaks in long-term care facilities, Oregon and Minnesota, USA, 2002-2009. Emerg Infect Dis 18:873-876. DOI 10.3201/eid1805.111843

Liu X, Jahuira H, Gilman RH, Alva A, Cabrera L, Okamoto M, Xu H, Windle HJ, Kelleher D, Varela M, Verastegui M, Calderon M, Sanchez G, Sarabia V, Ballard SB, Bern C, Mayta H, Crabtree JE, Cama V, Saito M, and Oshitani H. 2016. Etiological Role and Repeated Infections of Sapovirus among Children Aged Less than 2 Years in a Cohort Study in a Peri-urban Community of Peru. J Clin Microbiol 54:1598-1604. DOI $10.1128 / \mathrm{jcm} .03133-15$

Liu X, Yamamoto D, Saito M, Imagawa T, Ablola A, Tandoc AO, 3rd, Segubre-Mercado E, Lupisan SP, Okamoto M, Furuse Y, Saito M, and Oshitani H. 2015. Molecular detection and characterization of sapovirus in hospitalized children with acute gastroenteritis in the Philippines. J Clin Virol 68:83-88. DOI 10.1016/j.jcv.2015.05.001

Lole KS, Bollinger RC, Paranjape RS, Gadkari D, Kulkarni SS, Novak NG, Ingersoll R, Sheppard HW, and Ray SC. 1999. Full-length human immunodeficiency virus type 1 genomes from subtype C-infected seroconverters in India, with evidence of intersubtype recombination. $J$ Virol 73:152-160.

Lu L, Zhong H, Xu M, Su L, Cao L, Dong N, and Xu J. 2014. Molecular epidemiology of human calicivirus infections in children with acute diarrhea in Shanghai: a retrospective comparison between inpatients and outpatients treated between 2006 and 2011. Arch Virol 159:1613-1621. DOI 10.1007/s00705-013-1881-6 
383 Magwalivha M, Kabue JP, Traore AN, and Potgieter N. 2018. Prevalence of Human Sapovirus

384 in Low and Middle Income Countries. Adv Virol 2018:5986549. DOI

385 10.1155/2018/5986549. eCollection 2018

386

387

388

389

390

391

392

393

394

395

396

397

398

399

400

401

402

403

Martin DP, Murrell B, Golden M, Khoosal A, and Muhire B. 2015. RDP4: Detection and analysis of recombination patterns in virus genomes. Virus Evol 1:vev003. DOI $10.1093 / \mathrm{ve} / \mathrm{vev} 003$

Martinez N, Espul C, Cuello H, Zhong W, Jiang X, Matson DO, and Berke T. 2002. Sequence diversity of human caliciviruses recovered from children with diarrhea in Mendoza, Argentina, 1995-1998. J Med Virol 67:289-298. DOI 10.1002/jmv.2220

Murray TY, Nadan S, Page NA, and Taylor MB. 2016. Diverse sapovirus genotypes identified in children hospitalised with gastroenteritis in selected regions of South Africa. J Clin Virol 76:24-29. DOI 10.1016/j.jcv.2016.01.003

Neo FJX, Loh JJP, Ting P, Yeo WX, Gao CQH, Lee VJM, Tan BH, and Ng CG. 2017. Outbreak of caliciviruses in the Singapore military, 2015. BMC Infect Dis 17(1):719. DOI $10.1186 / \mathrm{s} 12879-017-2821-\mathrm{y}$

Nguyen TA, Hoang L, Pham le D, Hoang KT, Okitsu S, Mizuguchi M, and Ushijima H. 2008. Norovirus and sapovirus infections among children with acute gastroenteritis in Ho Chi Minh City during 2005-2006. J Trop Pediatr 54:102-113. DOI 10.1093/tropej/fmm096

Oka T, Lu Z, Phan T, Delwart EL, Saif LJ, and Wang Q. 2016. Genetic Characterization and Classification of Human and Animal Sapoviruses. PLoS One 11:e0156373. DOI 10.1371/journal.pone.0156373 
404 Oka T, Mori K, Iritani N, Harada S, Ueki Y, Iizuka S, Mise K, Murakami K, Wakita T, and 405 Katayama K. 2012. Human sapovirus classification based on complete capsid nucleotide 406 sequences. Arch Virol 157:349-352. DOI 10.1007/s00705-011-1161-2

407 Oka T, Wang Q, Katayama K, and Saif LJ. 2015. Comprehensive review of human sapoviruses.

408 Clin Microbiol Rev 28:32-53. DOI 10.1128/cmr.00011-14

409 Phan TG, Yan H, Khamrin P, Quang TD, Dey SK, Yagyu F, Okitsu S, Muller WE, and Ushijima 410 H. 2006. Novel intragenotype recombination in sapovirus. Clin Lab 52:363-366.

411

412

413

414

415

416

417

418

419

420

421

422

423

424

425

Ren Z, Kong Y, Wang J, Wang Q, Huang A, and Xu H. 2013. Etiological study of enteric viruses and the genetic diversity of norovirus, sapovirus, adenovirus, and astrovirus in children with diarrhea in Chongqing, China. BMC Infect Dis 13:412. DOI 10.1186/14712334-13-412

Sala MR, Broner S, Moreno A, Arias C, Godoy P, Minguell S, Martinez A, Torner N, Bartolome R, de Simon M, Guix S, and Dominguez A. 2014. Cases of acute gastroenteritis due to calicivirus in outbreaks: clinical differences by age and aetiological agent. Clin Microbiol Infect 20:793-798. DOI 10.1111/1469-0691.12522

Supadej K, Khamrin P, Kumthip K, Malasao R, Chaimongkol N, Saito M, Oshitani H, Ushijima H, and Maneekarn N. 2019. Distribution of norovirus and sapovirus genotypes with emergence of NoV GII.P16/GII.2 recombinant strains in Chiang Mai, Thailand. J Med Virol 91:215-224. DOI 10.1002/jmv.25261

Thongprachum A, Takanashi S, Kalesaran AF, Okitsu S, Mizuguchi M, Hayakawa S, Ushijima H. 2015. Four-year study of viruses that cause diarrhea in Japanese pediatric outpatients. J Med Virol 87(7):1141-1148. DOI 10.1002/jmv.24155 
426 Torner N, Martinez A, Broner S, Moreno A, Camps N, and Dominguez A. 2016. Epidemiology

427 of Acute Gastroenteritis Outbreaks Caused by Human Calicivirus (Norovirus and

428 Sapovirus) in Catalonia: A Two Year Prospective Study, 2010-2011. PLoS One

429 11:e0152503. DOI 10.1371/journal.pone.0152503

430 Trang NV, Luan le T, Kim-Anh le T, Hau VT, Nhung le TH, Phasuk P, Setrabutr O, Shirley H,

431 Vinje J, Anh DD, and Mason CJ. 2012. Detection and molecular characterization of

432 noroviruses and sapoviruses in children admitted to hospital with acute gastroenteritis in

433 Vietnam. J Med Virol 84:290-297. DOI 10.1002/jmv.23185

434 Varela MF, Rivadulla E, Lema A, Romalde JL. 2019. Human Sapovirus among Outpatients with 435 Acute Gastroenteritis in Spain: A One-Year Study. Viruses 11(2). pii: E144. DOI

$436 \quad 10.3390 / \mathrm{v} 11020144$

437 Worobey M, and Holmes EC. 1999. Evolutionary aspects of recombination in RNA viruses. $J$

438 Gen Virol 80 ( Pt 10):2535-2543. DOI 10.1099/0022-1317-80-10-2535

439 Xue L, Cai W, Gao J, Jiang Y, Wu H, Zhang L, Zuo Y, Dong R, Pang R, Zeng H, Wu S, Wang

440 J, Zhang J, and Wu Q. 2019. Genome characteristics and molecular evolution of the

441 human sapovirus variant GII.8. Infect Genet Evol 73:362-367. DOI

$442 \quad$ 10.1016/j.meegid.2019.05.017

443 Yan H, Yagyu F, Okitsu S, Nishio O, and Ushijima H. 2003. Detection of norovirus (GI, GII),

444 Sapovirus and astrovirus in fecal samples using reverse transcription single-round

445 multiplex PCR. J Virol Methods 114:37-44.

446 Yinda CK, Conceicao-Neto N, Zeller M, Heylen E, Maes P, Ghogomu SM, Van Ranst M, and

447 Matthijnssens J. 2017. Novel highly divergent sapoviruses detected by metagenomics 


\section{Table 1 (on next page)}

Oligonucleotide primers used for amplification of SaV capsid and polymerase genes. 


\begin{tabular}{llllll}
\hline Primer & Sequence (5'-3') & Direction & Target gene & $\begin{array}{l}\text { Position in SaV genome } \\
\text { (Accession no. X86560) }\end{array}$ & Reference \\
\hline SLV5317 & CTCGCCACCTACRAWGCBTGGTT & Forward & VP1 & $5083-5105$ & (Yan et al. 2003) \\
SLV5749 & CGGRCYTCAAAVSTACCBCCCCA & Reverse & VP1 & $5494-5516$ & (Yan et al. 2003) \\
Sapp36 & GTTGCTGTTGGCATTAACA & Forward & RdRp & $4273-4291$ & (Berke et al. 1997) \\
Sapp128 & GATTACACCAAATGGGATTCCAC & Forward & RdRp & $4354-4376$ & (Martinez et al. 1998) \\
SaV1245R & CCCTCCATYTCAAACACTA & Reverse & RdRp & $5159-5177$ & (Harada et al. 2013) \\
SV-r-c & GCATTGTAGGTGGCGAGAGCC & Reverse & RdRp & $5079-5099$ & (Homma et al. 2001)
\end{tabular}

2

3

4

5 


\section{Table 2 (on next page)}

Prevalence and genotype distribution of sapovirus detected in children with acute gastroenteritis in Chiang Mai, Thailand from 2010 to 2018. 


\begin{tabular}{|c|c|c|c|c|c|c|c|c|c|}
\hline \multirow[t]{2}{*}{ Years } & \multirow{2}{*}{$\begin{array}{c}\text { Total } \\
\text { samples }\end{array}$} & \multirow{2}{*}{$\begin{array}{c}\text { Positive } \\
\text { samples } \\
(\%)\end{array}$} & \multicolumn{7}{|c|}{ SaV genotypes } \\
\hline & & & GI.1 & GI.2 & GI.5 & GII.1 & GII.5 & GIV.1 & GII.1/GII.4 \\
\hline 2010 & 109 & $2(1.8)$ & 2 & - & - & - & - & - & - \\
\hline 2011 & 302 & 0 & - & - & - & - & - & - & - \\
\hline 2012 & 341 & $2(0.6)$ & - & - & - & - & - & 1 & 1 \\
\hline 2013 & 280 & $4(1.4)$ & 2 & - & - & 1 & - & - & 1 \\
\hline 2014 & 268 & 0 & - & - & - & - & - & - & - \\
\hline 2015 & 335 & $6(1.8)$ & 3 & 3 & - & - & - & - & - \\
\hline 2016 & 508 & $11(2.2)$ & 6 & 3 & - & - & 2 & - & - \\
\hline 2017 & 278 & $7(2.5)$ & 3 & - & - & 2 & - & - & 2 \\
\hline 2018 & 636 & $18(2.8)$ & 4 & 1 & 1 & 5 & 6 & 1 & - \\
\hline 9 years & 3057 & $50(1.6)$ & 20 & 7 & 1 & 8 & 8 & 2 & 4 \\
\hline
\end{tabular}

3

4

5

6

7 


\section{Table 3 (on next page)}

General information of patients and genotypes of sapovirus based on polymerase and capsid nucleotide sequences. 


\begin{tabular}{|c|c|c|c|c|c|c|}
\hline Sample ID & Collection date & Age & Gender & $\begin{array}{c}\text { RdRp } \\
\text { genotype }\end{array}$ & $\begin{array}{c}\text { VP1 } \\
\text { genotype }\end{array}$ & Mix-infection \\
\hline CMH-N018-10 & 12-Dec-2010 & - & Female & GI.1 & GI.1 & - \\
\hline CMH-S050-10 & 14-Dec-2010 & - & Female & GI.1 & GI.1 & - \\
\hline CMH-N061-12 & 11-Feb-2012 & - & Female & GIV.1 & GIV.1 & - \\
\hline CMH-N145-12 & 10-Aug-2012 & - & Male & GII.1 & GII.4 & - \\
\hline CMH-S004-13 & Jan-2013 & - & Male & GI.1 & GI.1 & - \\
\hline CMH-S034-13 & Apr-2013 & - & Female & GII.1 & GII.1 & Rotavirus \\
\hline CMH-N021-13 & 02-Мay-2013 & - & Male & GII.1 & GII.4 & Rotavirus \\
\hline CMH-N131-13 & $03-O c t-2013$ & - & Female & GI.1 & GI.1 & - \\
\hline CMH-S152-15 & 16-Jun-2015 & 28 months & Male & GI.2 & GI.2 & - \\
\hline CMH-S166-15 & 13-Aug-2015 & 35 months & Female & GI.1 & GI.1 & - \\
\hline CMH-S252-15 & 20-Dec-2015 & 54 months & Female & GI.2 & GI.2 & - \\
\hline CMH-S254-15 & 31-Dec-2015 & 32 months & Female & GI.2 & GI.2 & Norovirus \\
\hline CMH-N006-15 & 09 -Jul-2015 & 7 months & Male & GI.1 & GI.1 & Adenovirus \\
\hline CMH-N007-15 & 09 -Jul-2015 & 9 months & Male & GI.1 & GI.1 & Parechovirus \\
\hline CMH-S108-16 & 29-Mar-2016 & 20 months & Female & GI.2 & GI.2 & - \\
\hline CMH-S198-16 & 11-Aug-2016 & 32 months & Male & GI.1 & GI.1 & Enterovirus \\
\hline CMH-S229-16 & 12-Sep-2016 & 20 months & Male & GI.1 & GI.1 & - \\
\hline CMH-ST004-16 & 11-Jan-2016 & 27 months & Female & GI.2 & GI.2 & - \\
\hline CMH-ST016-16 & 02-Feb-2016 & 22 months & Male & GI.1 & GI.1 & - \\
\hline CMH-ST029-16 & 07-Feb-2016 & 143 months & Female & GI.2 & GI.2 & - \\
\hline CMH-ST090-16 & 21-Mar-2016 & 26 months & Female & GI.1 & GI.1 & Norovirus \\
\hline CMH-ST091-16 & 21-Mar-2016 & 8 months & Female & GII.5 & GII.5 & - \\
\hline CMH-ST095-16 & 23-Mar-2016 & 8 months & Female & GII.5 & GII.5 & - \\
\hline CMH-ST163-16 & 13-Aug-2016 & 24 months & Female & GI.1 & GI.1 & Parechovirus \\
\hline CMH-ST199-16 & 12-Dec-2016 & 27 months & Male & GI.1 & GI.1 & Parechovirus \\
\hline CMH-S003-17 & 22-Feb-2017 & 43 months & Male & GI.1 & GI.1 & Astrovirus \\
\hline CMH-S023-17 & 04-Apr-2017 & 34 months & Male & GI.1 & GI.1 & - \\
\hline CMH-S050-17 & 16-Jun-2017 & 16 months & Female & GII.1 & GII.4 & - \\
\hline CMH-S057-17 & 21-Jun-2017 & 22 months & Female & GII.1 & GII.4 & - \\
\hline CMH-S089-17 & 21-Jun-2017 & 12 months & Female & GI.1 & GI.1 & Enterovirus \\
\hline CMH-S120-17 & 21-Nov-2017 & 16 months & Male & GII.1 & GII.1 & - \\
\hline CMH-ST028-17 & 09-May-2017 & 24 months & Male & GII.1 & GII.1 & - \\
\hline CMH-N028-18 & 02-Feb-2018 & 16 months & Female & GIV.1 & GIV.1 & Rotavirus, Parechovirus \\
\hline CMH-N061-18 & 18-Feb-2018 & 26 months & Female & GI.5 & GI.5 & Rotavirus \\
\hline CMH-N091-18 & 24-Jun-2018 & 18 months & Male & GII.1 & GII.1 & - \\
\hline CMH-N104-18 & 10-Sep-2018 & 11 months & Male & GII.1 & GII.1 & - \\
\hline CMH-R031-18 & 21-Oct-2018 & 24 months & Female & GI.1 & GI.1 & - \\
\hline CMH-R076-18 & 21-Oct-2018 & 24 months & Male & GI.2 & GI.2 & - \\
\hline CMH-R089-18 & 21-Oct-2018 & 43 months & Male & GII.5 & GII.5 & - \\
\hline CMH-R140-18 & 21-Oct-2018 & 12 months & Female & GII.1 & GII.1 & - \\
\hline CMH-S174-18 & 21-Nov-2018 & 48 months & Male & GI.1 & GI.1 & - \\
\hline CMH-S175-18 & 21-Nov-2018 & 31 months & Male & GII.1 & GII.1 & - \\
\hline CMH-ST097-18 & 16-Apr-2018 & 25 months & Male & GI.1 & GI.1 & - \\
\hline CMH-ST169-18 & 27-Jun-2018 & 12 months & Male & GI.1 & GI.1 & - \\
\hline CMH-ST189-18 & 27-Jul-2018 & 12 months & Male & GII.1 & GII.1 & - \\
\hline CMH-ST202-18 & 15-Aug-2018 & 20 months & Male & GII.5 & GII.5 & - \\
\hline CMH-ST207-18 & 17-Aug-2018 & 22 months & Male & GII.5 & GII.5 & - \\
\hline CMH-ST247-18 & 10-Nov-2018 & 44 months & Male & GII.5 & GII.5 & - \\
\hline CMH-ST266-18 & 15-Dec-2018 & 24 months & Female & - & GII.5 & - \\
\hline CMH-ST270-18 & 29-Dec-2018 & 32 months & Female & GII.5 & GII.5 & - \\
\hline
\end{tabular}

1 The highlighted samples are the ones in which recombinant sapoviruses were found. 


\section{Figure 1}

Phylogenetic tree of the partial capsid gene sequences (296 nucleotides).

Fifty SaV strains detected in this study are indicated by black circle (non-recombinant strains) and red triangle (recombinant strains). The evolutionary history was inferred by using the Maximum Likelihood method based on the Kimura 2-parameter model. Scale bar indicates nucleotide substitutions per site and bootstrap values $(>80)$ are indicated for the corresponding nodes. 


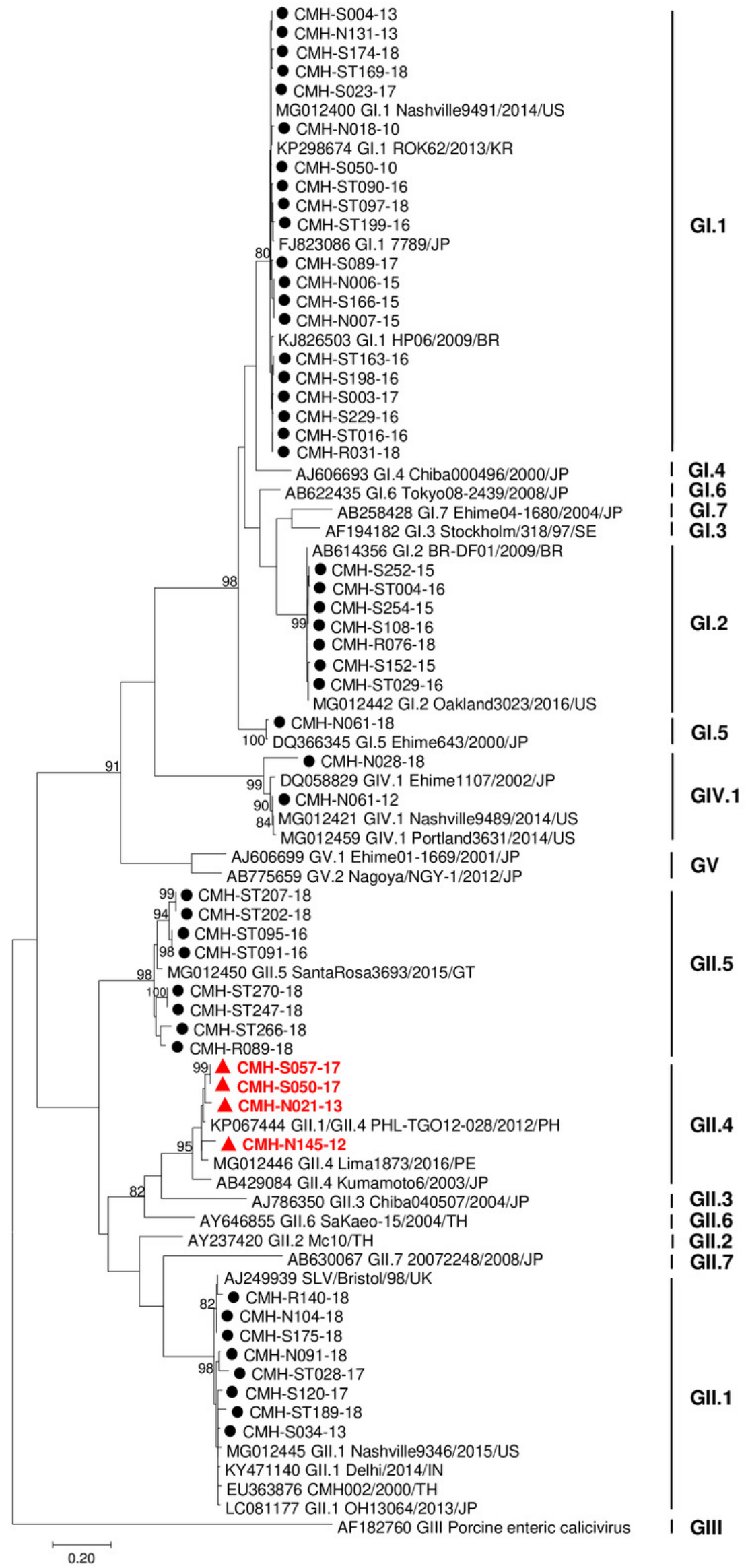


Figure 2

Phylogenetic tree of the partial RdRp gene sequences (745 nucleotides).

Forty-nine SaV strains detected in this study are indicated by black circle (non-recombinant strains) and blue triangle (recombinant strains). The evolutionary history was inferred by using the Maximum Likelihood method based on the General Time Reversible model. Scale bar indicates nucleotide substitutions per site and bootstrap values $(>80)$ are indicated for the corresponding nodes. 


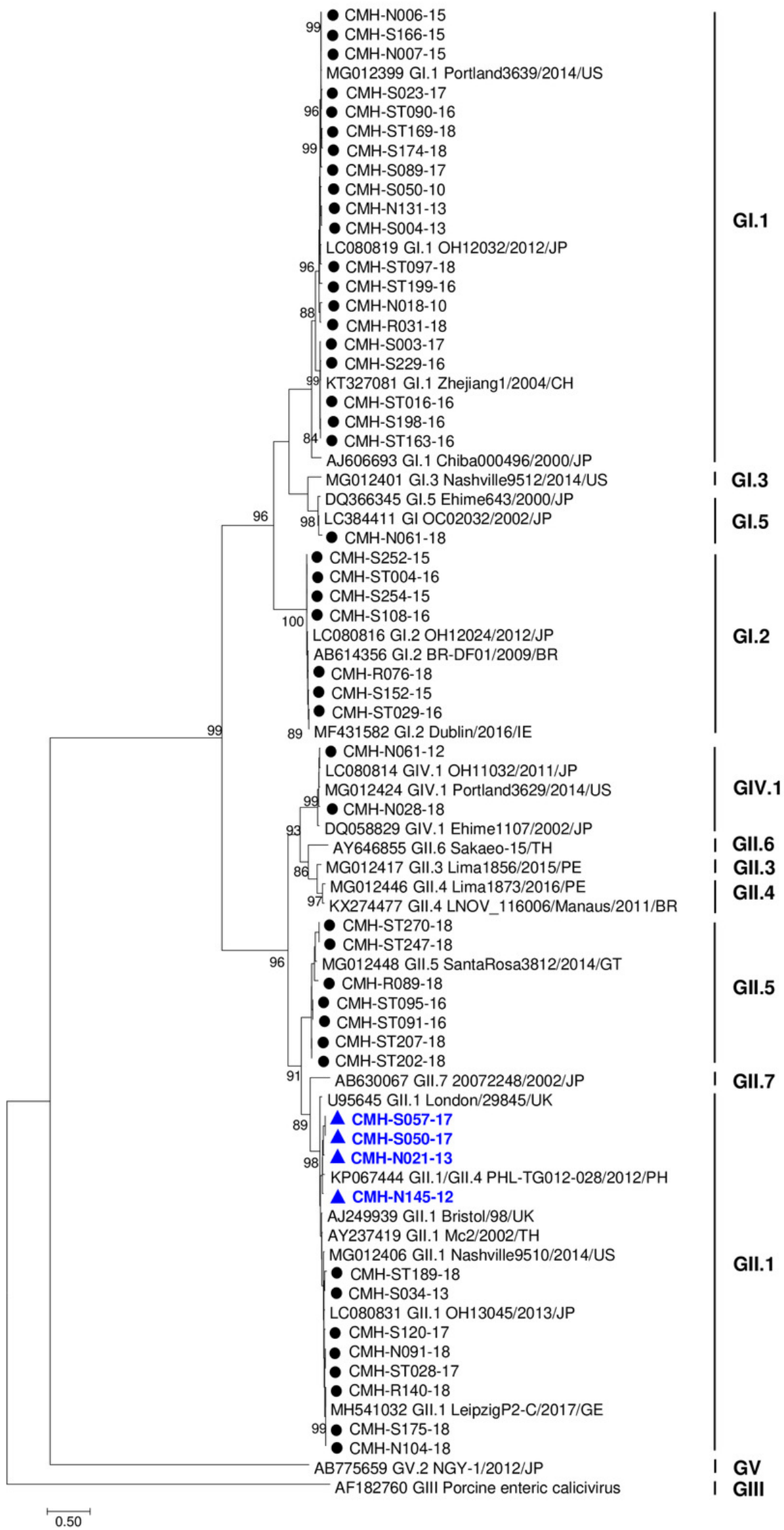


Figure 3

The similarity plot of four SaV recombinant strains was constructed using SimPlot software.

The similarity score of 200 nucleotides sliding window and 20 site step were used. The junction of RdRp and capsid sequences of SaV recombinant strains (1055 nucleotides) were plotted against the nucleotide sequences of the reference strains, GII.1/Bistol/98/UK and GIl.4/Lima1873/2016/PE. The x-axis represents the nucleotide position and the $y$-axis indicates the similarity between the query strain and the reference strains.
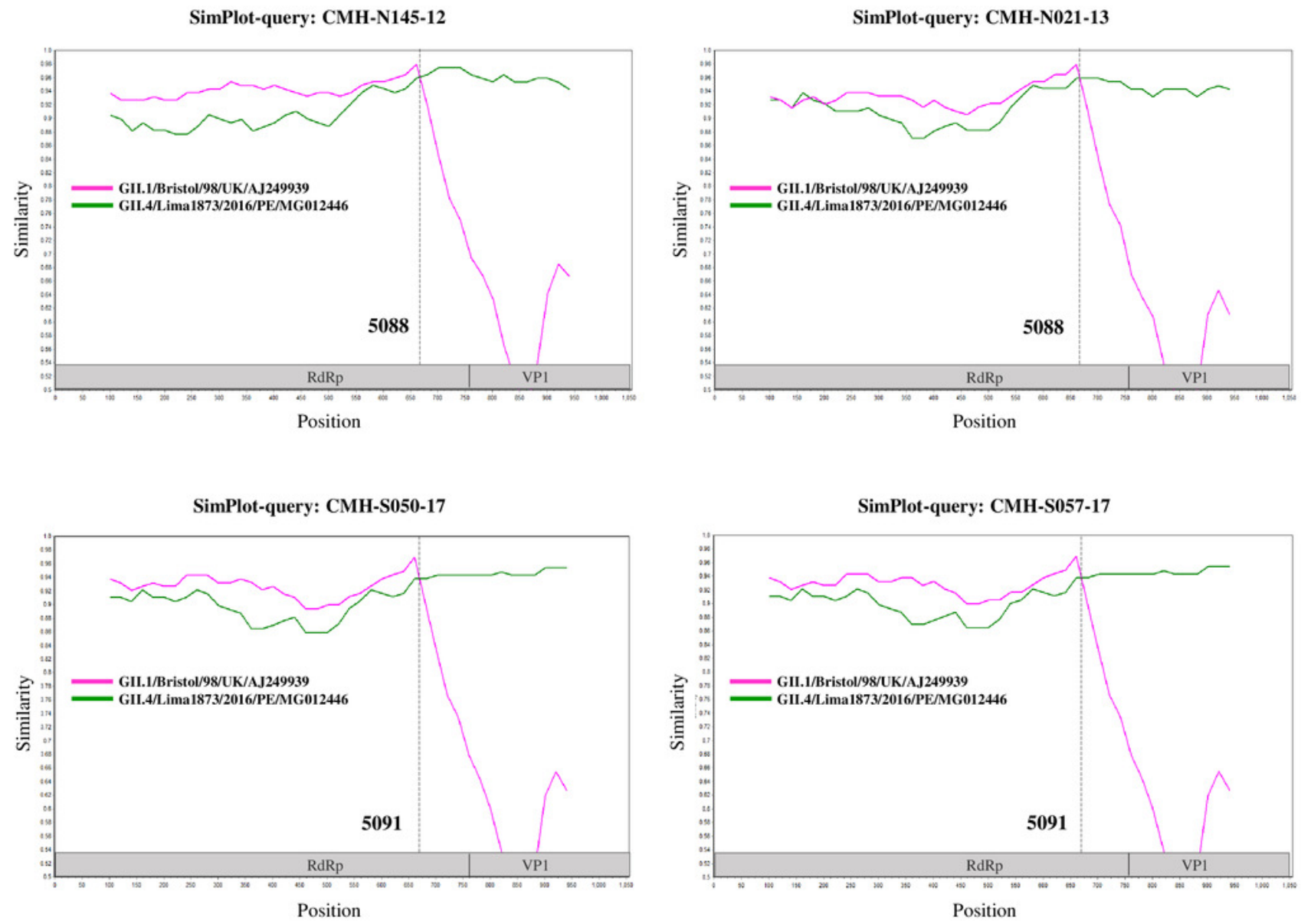
Figure 4

Distribution of SaV genotypes detected in different age groups of patients. 


\section{Number of patients}

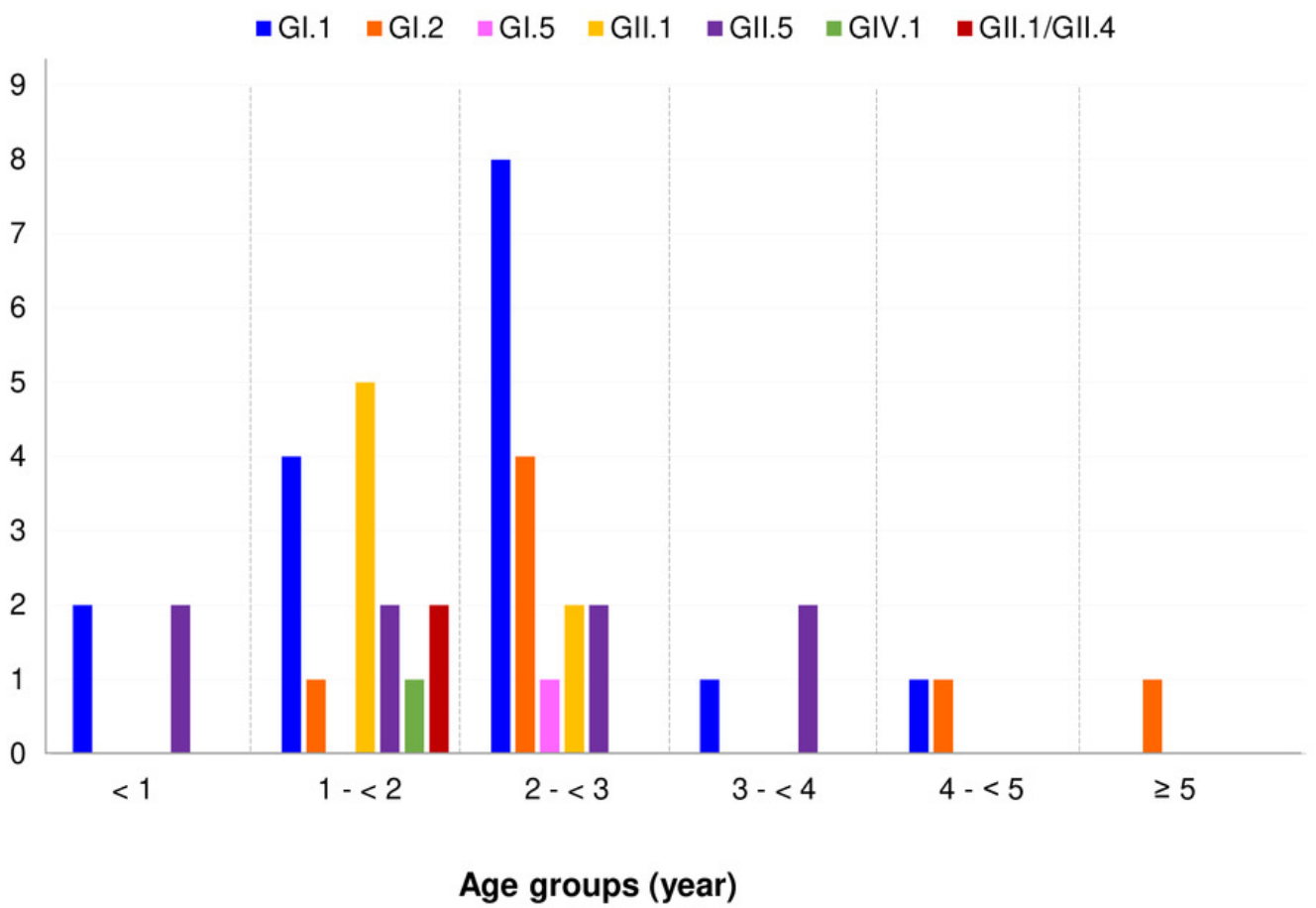

